\title{
The balance of autonomous and centralized control in scheduling problems
}

\author{
Henning Blunck ${ }^{1}$, Dieter Armbruster ${ }^{2}$, Julia Bendul ${ }^{*}{ }^{*}$ and Marc-Thorsten Hütt ${ }^{3}$
}

${ }^{*}$ Correspondence:

bendul@scm.rwth-aachen.de

${ }^{1}$ RWTH Aachen University, School of

Management \& Economics,

Kackertstr. 8, 52062 Aachen,

Germany

Full list of author information is

available at the end of the article

\begin{abstract}
The scheduling of processes in a network is a core logistic challenge with a multitude of applications in our complex industrialized world. Often, scheduling decisions are based on incomplete and unreliable information. Here, a simple rule of 'more information, better decisions' may no longer hold and heuristics balancing global and local information, or centralized and autonomous control, may yield better performance. So far, only anecdotal evidence for the potential benefit of autonomous control in scheduling exists. Here, we explore this hypothesis within a minimal model derived from scheduling principles and the phenomenology of dynamical processes on graphs. In this model, centralized and autonomous control can be represented and quantitatively assessed, performance is well defined and problem complexity can be varied.

Our model shows that a balance of centralized and autonomous control can enhance the performance in networks of decision-making entities. The mechanistic insight gained from the model also reveals the limitations of hybrid control setups: We find that communication at a high hierarchy level can give an advantage to centralized control. Counter-intuitively, it arises not from a higher degree of coordination and quicker convergence towards a common solution, but rather from an accelerated sampling of candidate choices leading to a measurable increase in information flow from higher to lower hierarchical levels. Our study allows us to formulate a new view of autonomous control in industrial production and derive a set of suggestions with the potential to enhance performance under realistic conditions of scheduling heuristics of jobs in a production process.
\end{abstract}

Keywords: Distributed decision making, Complex networks, Graph coloring dynamics, Industrial production, Industry 4.0

\section{Background}

Diverse real-world organizational challenges can be mapped onto the abstract problem of scheduling events on a network (Pinedo 2012). The corresponding networks could be towns connected by highways (Rosvall et al. 2005), machines connected by material flows (Becker et al. 2011) or routers connected by internet connections (Vázquez et al. 2002).

In an idealized world, with infinite computational resources (in particular a negligible processing time of information), an absolute knowledge about the functioning of the system (leading to optimal decision algorithms), and in the absence of noise and uncertainty in the data, the quality of a decision will necessarily increase with the amount of

(c) The Author(s). 2018 Open Access This article is distributed under the terms of the Creative Commons Attribution 4.0 International License (http://creativecommons.org/licenses/by/4.0/), which permits unrestricted use, distribution, and reproduction in any medium, provided you give appropriate credit to the original author(s) and the source, provide a link to the Creative Commons license, and indicate if changes were made. 
information included. In real-world situations, however, rapid decisions need to be made based on limited and occasionally unreliable information in systems, whose mechanistic rules of organization and functioning are partially unknown (Yang et al. 2007; Mula et al. 2006). In industrial production, autonomous control is perceived as a promising approach to achieve robustness against the increasing dynamical and structural complexity of production networks (Scholz-Reiter et al. 2009).

The general picture is the following: A decision requires evaluating the transition from one system state to another. Compared to evaluating global information, the combinatorial explosion of decision complexity is dramatically reduced by processing local information only. The gain in processing speed is generally considered the main advantage of autonomous control. A more subtle effect is that distal information can be systematically wrong or outdated due to the longer transmission time, leading to an increase in erroneous decisions, as they are based on essentially random information. This is particularly fascinating when the deterioration of distal information arises from the decision process itself (i.e. from the updates / decisions occurring at distal system sites), linking the topic of local decisions to the general framework of emergent collective behaviors and self-organization (Moreira et al. 2004a; Marr and Hütt 2006; Tang et al. 2014).

Evidence for global behavior arising from local decisions (or "autonomous control") has been provided for a multitude of applications, ranging from how social patterns arise (fads, fashions, youtube videos 'going viral') from localized conformity (Bikhchandani et al. 1992) to how stomatal cells in plant leaves compute their optimal opening state in a distributed fashion (Peak et al. 2004) and vehicle flow through bottlenecks (Lämmer and Helbing 2008).

The highly aggregated question of a benefit of autonomous control is situated at the interface of algorithmics, decision theory, logistics and systems science. It is encountered in a myriad of application scenarios - ranging from biology (Peak et al. 2004; Mott and Peak 2006) and social sciences (Schreyögg and Sydow 2010; Sales-Pardo et al. 2013) to information and communication technologies (Moreira et al. 2004a; Tang et al. 2014) and industrial production (Trentesaux 2009; Duffie et al. 2013).

In production networks, relevant information for centralized decision making is either not available at all or only after a time delay. Autonomous control strategies seem promising to overcome the related problems: Decentralized coordination of intelligent logistics objects (parts, orders, machines etc.) and the adaptive routing through the manufacturing and assembly system can positively impact logistics performance of industrial production (Scholz-Reiter and Freitag 2007; Scholz-Reiter et al. 2009). To achieve selforganization and emergent behavior, the logistic objects are capable to process local information, to decide and to communicate autonomously and to flexibly react on dynamic framework conditions and changing as well as conflicting target dimensions (Scholz-Reiter et al. 2005).

In Philipp et al. (2006); Windt et al. (2008) and Scholz-Reiter et al. (2009) it has been hypothesized that, under rather general assumptions about the nature of the system and the decision process, a balance of centralized and autonomous control yields the best systemic performance and that the performance gain from combining centralized and decentralized decision strategies is higher for a larger complexity of the decision task (see also Zambrano Rey et al. (2014)). This hypothesis, formulated with the framework of production logistics, has lead to an avalanche of research work in that field (see, e.g., 
(Duffie et al. 2013; Scholz-Reiter et al. 2010; Duffie 2008; Hongler et al. 2010; Schuh et al. 2014)).

Ever clearer links between network topology and the dynamical performance of supply, distribution and manufacturing networks have emerged over the last years (see, e.g., (Meyer et al. 2015; Perera et al. 2017; Matous and Todo 2017)).

Abstract, minimal process models provide a suitable formal framework for addressing such universal dynamical properties of networks (see, Watts (2002); Müller-Linow et al. (2008a); Bornholdt (2005); de Menezes and Barabási (2004) for examples from diverse fields). A similar approach can be employed for acquiring a generic insight into the balance of local and global information (or autonomous vs. centralized control) in scheduling decisions on networks (Windt and Hütt 2010). Some cornerstones of our understanding of this situation have already been contributed by such minimal process models in the past:

In Moreira et al. (2004b) the loss of performance due to noise for a specific local decision task, density classification, is analyzed. The result is that high performance can be restored by inserting distal information (shortcuts, representing a form of centralized control) into the local neighborhoods (representing autonomous control). Studying cellular automata on graphs, in Marr and Hütt (2006) it has been shown that shortcuts can also serve as noise sources by inserting random input into homogeneous local patterns. Relatedly, in the forest-fire model (Drossel and Schwabl 1992) (a minimal model of selforganized criticality) shortcuts can functionally substitute random excitation probability, which is the control parameter of the critical state (Graham and Matthai 2003).

In order to study, how a balance of centralized and autonomous control affects the performance of a production system, we employ a minimal model of the scheduling of (production) events on a graph: Graph coloring, the process of placing colors on a graph, such that no same colors are linked, is an algorithmic problem directly associated with a formal scheduling problem. De Werra and Hertz (2015) note that Graph Coloring "may provide a natural tool for dealing with a variety of scheduling problems". Determining, for a given graph $G$, the smallest number of colors $\chi=\chi(G)$, for which such a coloring is possible (known as the graph's chromatic number), is an NP-hard problem (Karp 1972). The underlying notion of conflicts (represented as edges in a graph) and conflict resolution under constraints (represented by the limited number of colors $\chi$ ), makes the graph coloring problem an ideal minimal model for resource allocation problems. It has been explored in various application areas, such as packet switching in shared radio channels (Yeo et al. 2002), register allocation (Smith et al. 2004), and diverse other scheduling problems (Myszkowski 2008; Halldórsson et al. 2003; Pardalos et al. 1999; Marx 2004).

The model we explore, graph coloring dynamics (GCD) for a given chromatic number, has been previously used in a sociological study (Kearns et al. 2006) yielding fascinating empirical results on the solvability of different network architectures under local information. The idea to investigate not the minimal number of colors necessary to color a given graph, but the time and effort required for distributed decision-making entities (associated with graph vertices) to reach a conflict-free coloring, given a feasible amount of colors was initially discussed as an abstract model to study distributed constraint satisfaction problems (Fabiunke 1999). In GCD a node selects a color from a given list (of length $\chi$ ) based on the colors in the direct neighborhood. Color changes in the neighborhood can trigger a new selection of a color, which in turn affects the color selection of other 
nodes, until the graph is 'solved. GCD is thus a dynamical process on a graph operating on a discrete state space of $\chi$ elements. In addition to being an interesting test scenario for social coordination, GCD is also an excellent framework for assessing (independently of experiments) the scheduling performance of both, network architectures and decision heuristics, when formally encoding and parameterizing different solution strategies (Hadzhiev et al. 2009; Windt and Hütt 2010).

The remainder of the article is structured as follows: First, we reproduce the hypothesis of Philipp et al. (2006); Windt et al. (2008) about the balance of centralized and autonomous control, which leads us to a counter-intuitive role of centralized control. Next, we develop a mechanistic understanding by studying the time evolution of solution regimes on the graph. Lastly, we explore the real-life significance of the findings obtained with a schematic model by evaluating realistic performance indicators for industrial production. Details on our model, the evaluation of performance and the parameters used throughout the paper are provided in the "Methods" section.

\section{Results}

Performance as a function of autonomous control

Combining a regular (e.g., ring) architecture with a few randomly inserted shortcuts has become the most famous random graph model for small-world architectures, uniting high local clustering with a small average shortest path length (Watts and Strogatz 1998). Since the average path length decreases dramatically, already with a small percentage of shortcuts added, their insertion can be seen as a shift from a decentralized, local control to a more collective, centralized (even though disordered) control (Watts and Strogatz 1998).

From the viewpoint of production planning and control, variation of the amount of hierarchy in the network is typically considered a suitable way of structurally varying the balance between decentralized and centralized control - with highly hierarchical structures being associated with centralized, global control, while more spread-out, heterarchical structures are associated with decentralized, local control (Trentesaux 2009). Particularly when employing highly aggregated representations of dynamical processes, like cellular automata (Wolfram 1984), a variation of the update scheme (Marr and Hütt 2009) could provide an interesting alternative to the network strategies for varying the control type. Increasing neighborhood size could allow the transition towards ever more centralized control. In practice, however, such rule-based changes of the control type are not easy to implement: (1) update rules operating on different neighborhood sizes are difficult to compare (due to the different sizes of the underlying rule space); (2) in industrial production systems realistic networks typically have a few hundred nodes; in this case, the diameter of the graph is so small that when going from nearest neighbors to nextto-nearest neighbors in a node's update rule, already a substantial portion of the graph is covered ${ }^{1}$.

For our investigation we therefore employ a network architectural method for varying the balance of autonomous and centralized control. Our approach combines the key advantages of the previous approaches: the gradual variation of the control type as inherent in the shortcut-based approach and the association of centralized control with increased hierarchy known from production planning and control. We add 'leader nodes' with varying amounts of connectivity to the - mostly locally, heterarchically 
organized - main, ring-like part of the network. Figure 1 illustrates the setup of the system.

Summarizing, a GCD network $G=G\left(N, l, c, \chi, s, k_{L}, h\right)$ is then characterized by the number of ring nodes, $N$, the number of leader nodes, $l$, the number of connected neighbors, $c$, to the left and right of each node on the ring, the graph's chromatic number, $\chi$, the number of shortcuts $s$, as well as the number of links per leader node onto the main network, $k_{L}$, and a binary variable $h$ indicating whether the leader nodes are connected (i.e. they form a complete subgraph).

With the structural prerequisites of our model fixed, we define the dynamical model representing decision and scheduling processes. To test our hypotheses, we need an experimental setup, a conflict graph, that can expressed analytically as the result of a generating procedure. This is not only necessary to be able to change the degree of hierarchy in the system gradually and purposefully, but also to measure the problem-solving performance across multiple different "benchmark scenarios" (Hernando et al. 2016). Our model, GCD for a given chromatic number, is executed in the following way (Hadzhiev et al. 2009; Windt and Hütt 2010): Given an initial distribution of colors, nodes are scheduled for an update according to the 'channeled excitation' scheme from (Hadzhiev et al. 2009), where color changes in the neighborhood prompt an update. For such a color update of a node, the colors in the neighborhood are evaluated and the conflictminimizing color is picked. If the node already has the conflict-minimizing color, no update is performed (with probability $p$ ) or a random color is selected (with probability $1-p)$. The number of color changes $r_{C}(G)$ until the system converges to a fully solved

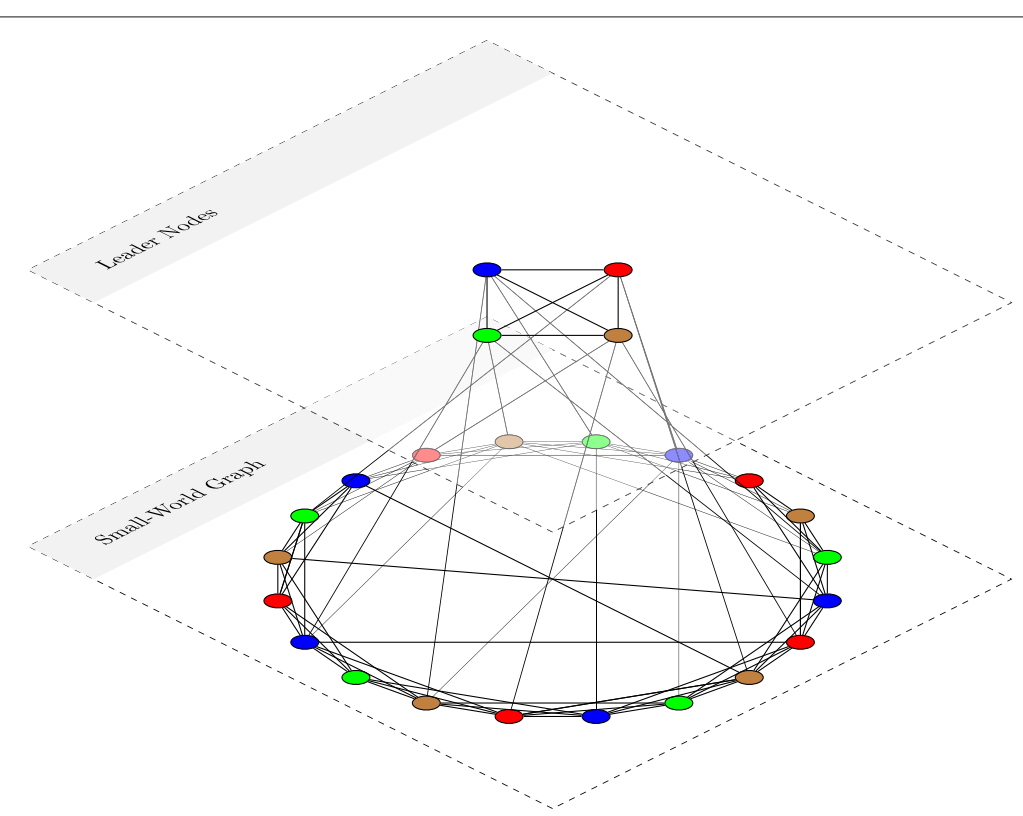

Fig. 1 Fully solved GCD network with $\chi=4$, based on a small-world graph with $N=20$ nodes and 12 randomly added shortcuts (lower level), enriched by four leader nodes that form a complete subgraph (upper level). Between both levels, a total of 12 links connect the leader nodes with the ring. This figure illustrates the general principle of the graph coloring problem - finding a distribution of colors such that no same colors are linked (conflict-free coloring) and finding the minimal number of colors (the graph's chromatic number) for which such a conflict-free coloring exists - as well as the distinction between 'ring nodes' and 'leader nodes' used throughout this investigation. For our investigation we assume the chromatic number to be given and study the convergence to a conflict-free coloring using dynamics generated by simple local update rules 
graph, averaged over many initial color distributions, is then measured. Details are given in the "Methods" section. Figure 1 gives an example of a successful coloring with four colors $(\chi=4)$.

In the following investigation, we will systematically vary the chromatic number $\chi$ (and, consequently, the coordination number $c$; see "Methods" section) ${ }^{2}$, as well as the number of links per leader node $k_{L}$, and measure the ability of the networks to solve the GCD problem. Since we are interested in the effect of network structure on the solution process we normalize $r_{C}(G)$ by the number of color changes required by a network with equal number of nodes and links (see "Methods" section), to remove the effect of increasing network connectivity investigated in Hadzhiev et al. (2009). We will consider a system where every leader node is connected to as many ring nodes as possible $\left(k_{L}=(\chi-1) / \chi\right.$. $N)$, to have $0 \%$ autonomous control and a system with unconnected leader nodes $\left(k_{L}=0\right)$ to exhibit $100 \%$ autonomous control.

Figure 2 shows the main result obtained in this numerical framework. Figure 2a reproduces precisely the salient features sketched in a speculative manner in Philipp et al. (2006); Windt et al. (2008); Zambrano Rey et al. (2014): Indeed, a balance of centralized and autonomous control leads to the highest gain in performance over the reference setting and the peak becomes more pronounced with increasing complexity of the computational problem (here: with an increasing chromatic number of the graph). With Fig. 2b, the numerical findings go beyond the hypotheses put forward before: For a higher level of coordination within centralized control (here: represented by the links among leader nodes) performance increases and the region of highest performance is shifted further towards centralized control (higher connectivity between leader nodes and ring nodes). The initial increase is in agreement with our intuition about the performance of the scheduling heuristics: An increase in centralized control increases the performance. The two counter-intuitive features of Fig. 2 are the subsequent decrease of performance at even higher centralized control and the dramatic qualitative change of this behavior, as soon as the leader nodes (representing centralized control or "upper management") are interconnected. For visual clarity, Fig. $2 b$ shows two representative curves (for unconnected and connected leader nodes) at fixed $\chi$. A 2D version of Fig. 2a is available as Additional file 1, together with the corresponding figure for connected leader nodes.

\section{Time evolution of solution regimes}

Our minimal model now allows us to go beyond the mere numerical observation of these effects and to understand the underlying organizational principles in more detail. To this end, we analyze the solution domains forming over time. A solution domain is a solved region in the graph, e.g. a sequence of nodes which are all in the same solution regime (see "Methods" section).

Figure 3a shows a space-time plot of the emergent solution domains, together with the time course of the number of color conflicts present in the graph. Qualitatively speaking, three dynamical phases can be seen. The first phase is characterized by a rapid decrease of color conflicts (which is a transient away from the random initial conditions). In the second phase, the system settles into a more systematic formation of solution domains, which co-exist and sometimes merge. The third, long phase is characterized by a very small number of color conflicts diffusing on the (ring) graph. Figure 3b shows the frequencies 

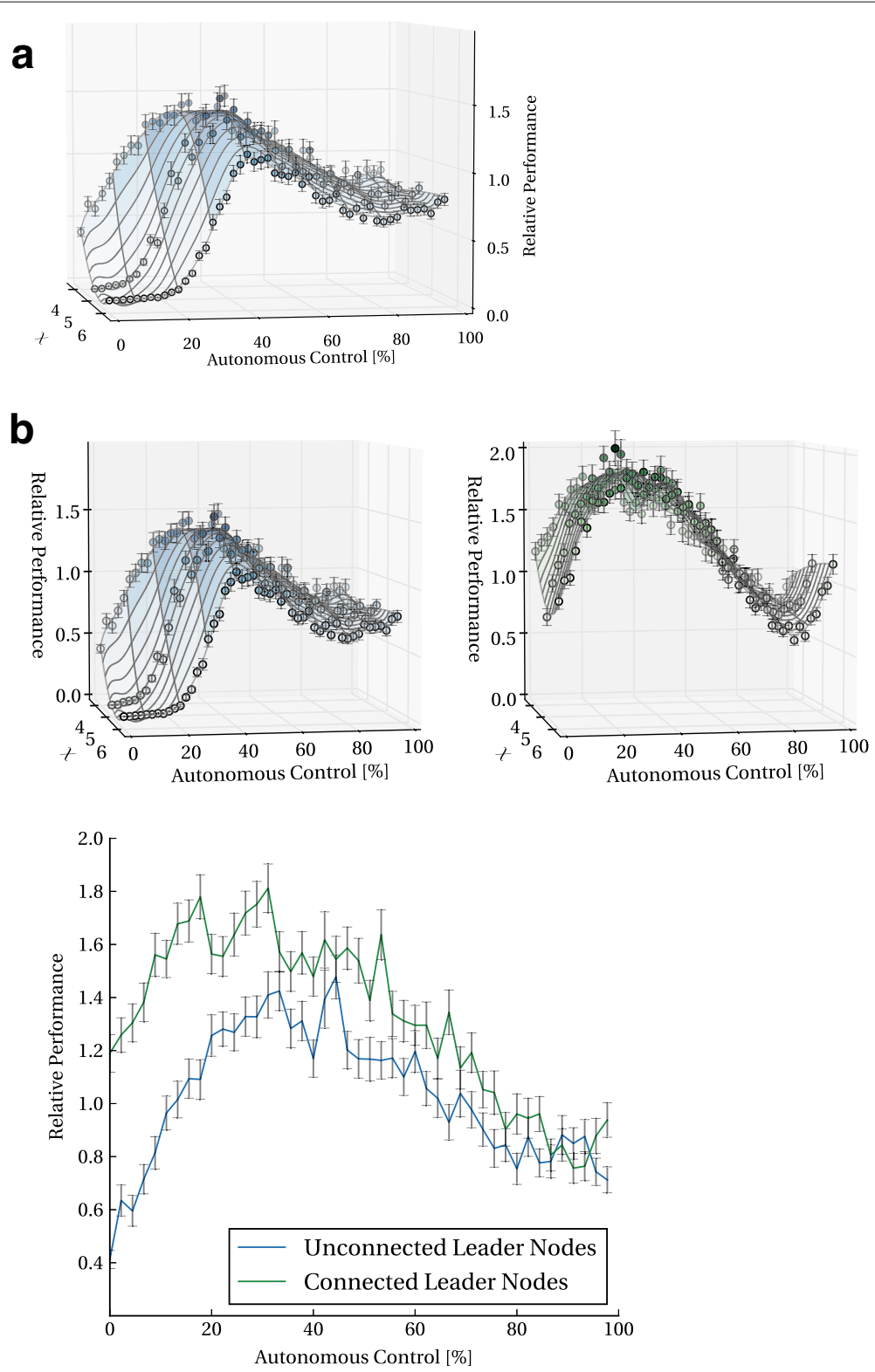

Fig. 2 Performance as a function of the level of autonomous control and the chromatic number $\chi(\mathbf{a})$ for unconnected leader nodes, (b) comparison between connected and unconnected leader nodes for $\chi=4$

of color changes for leader nodes and ring nodes for the last two of the phases discussed above both in the case of unconnected and of connected leader nodes.

Figure $3 \mathrm{~b}$ does not reveal any difference between connected and unconnected leader nodes. However, in both configurations we observe systematically higher frequencies of color changes for leader nodes compared to ring nodes ${ }^{3}$. On the basis of this observation, we will now investigate the information transmission from leader nodes to ring nodes and the functional role of these high-frequency leader nodes.

Formally for each node $i$, the three time series $x_{i}(t), s_{i}(t), f_{i}(t)$, denoting color, membership in a solution regime, and color change indicator, respectively, can be used to compute pair probabilities and the mutual information as described in the "Methods" section. 


\section{a}
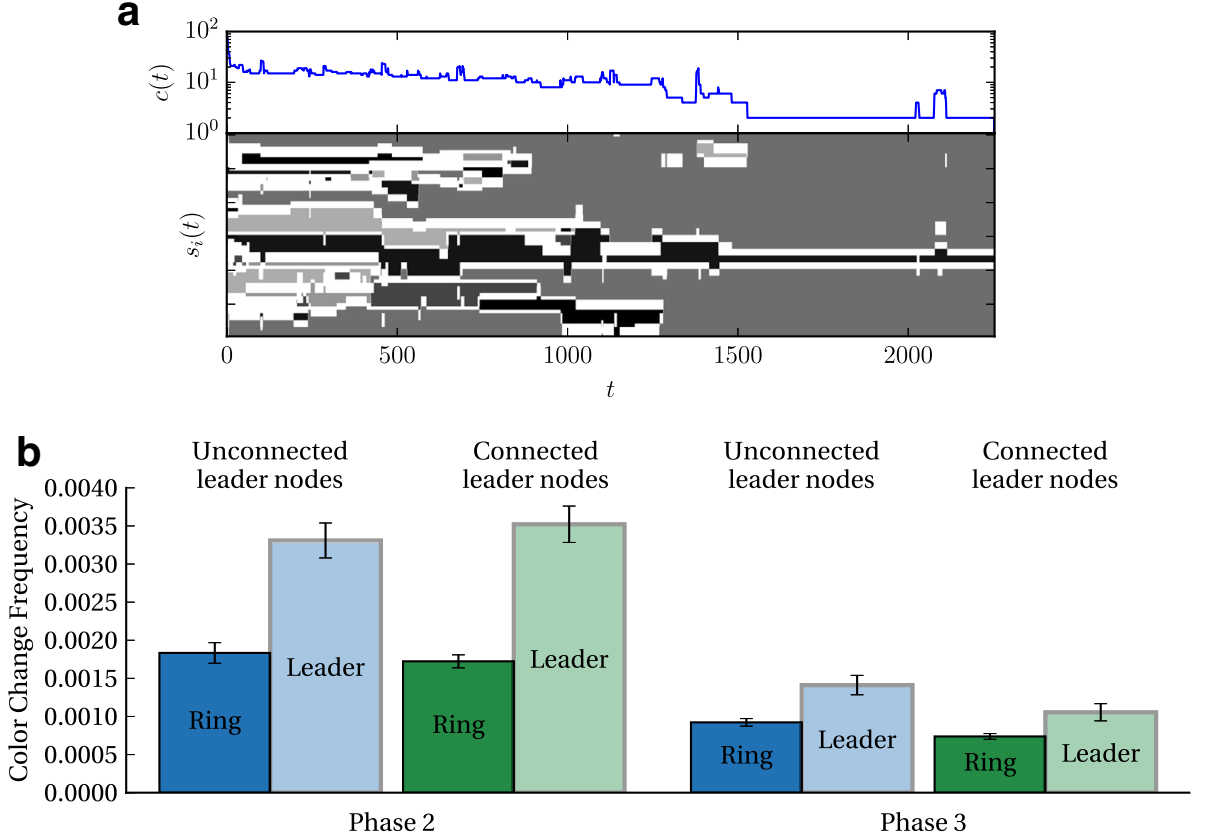

Fig. 3 Individual and collective node activity over time. a Exemplary domain evolution along the ring-nodes, together with the evolution of conflicts $c(t)$ in the graph (log-scale). Parameter settings are:

$N=60, \chi=4, s=30, k_{L}=20$ with no connection between leader nodes. $\mathbf{b}$ Color change frequencies over the last two phases of the solution process. Error bars show standard error of sample mean

Figure 4 reveals that connections among leader nodes enhance information transfer towards ring nodes when measured on the binary alphabet of whether a node is in a solution regimes. The communication among leader nodes consequently accelerates the process of solution regime formation and dissolution of local, globally incompatible regimes, rather than affecting the compatibilities of different solution regimes. This is confirmed by directly measuring the average number of incompatible solution regimes in both cases as well as by evaluating the information transfer on the level of colors (rather than solution regimes) (see Additional file 1).

\section{Noise-emitting leader-nodes}

The high color change frequencies of leader nodes, together with the enhanced information transfer from leader nodes to ring nodes, leads to the hypothesis that color changes in leader nodes drive the solution search process in the ring. This hypothesis can be further substantiated, when discussing the leader nodes as noise emitters in the solution process that can instigate ring nodes to leave local optima, similar to a distributed simulated annealing process (cf. (Arshad and Silaghi 2004)). In such metaheuristics, the probability that the search process leaves attained local minima decreases with time. The specific approach applied here is described in "Methods" section.

As shown in Fig. 5, the model variant with noise-emitting leader nodes reveals a similar behavior as the performance curves in Fig. 2b. The comparison of Fig. 5, together with the observations from Figs. 3 and 4, now provides a clear picture of the underlying mechanisms: 


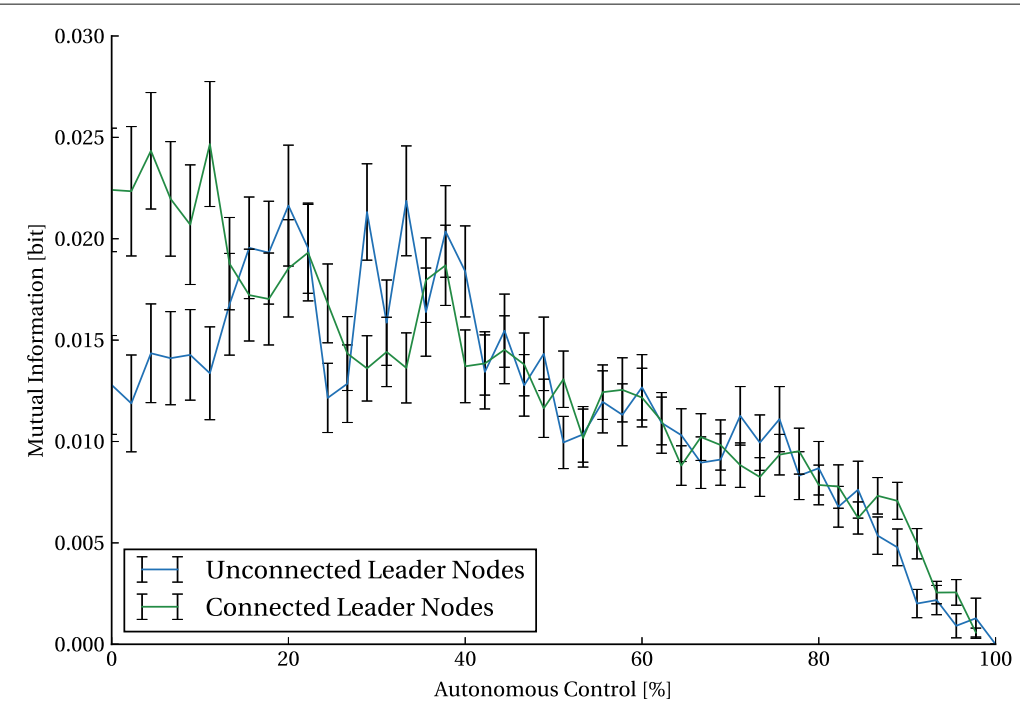

Fig. 4 Mutual information from leader to ring nodes based on solution regimes as a function of the degree of autonomous control

The initial increase of performance with increasing degree of the leader nodes can be mimicked by substituting the leader nodes by noise sources. The rapid color fluctuations of leader nodes insert enough random information into the ring that the system moves out of regional solutions, which are globally incompatible. The frequency of color changes of the leader nodes decreases with time (and decreasing color conflicts in the system), even though the frequency is still higher than for the ring nodes. The function of leader nodes is thus reminiscent of noise in simulated annealing. Indeed, simulated annealing strategies for graph coloring have been studied in Johnson et al. (1991).

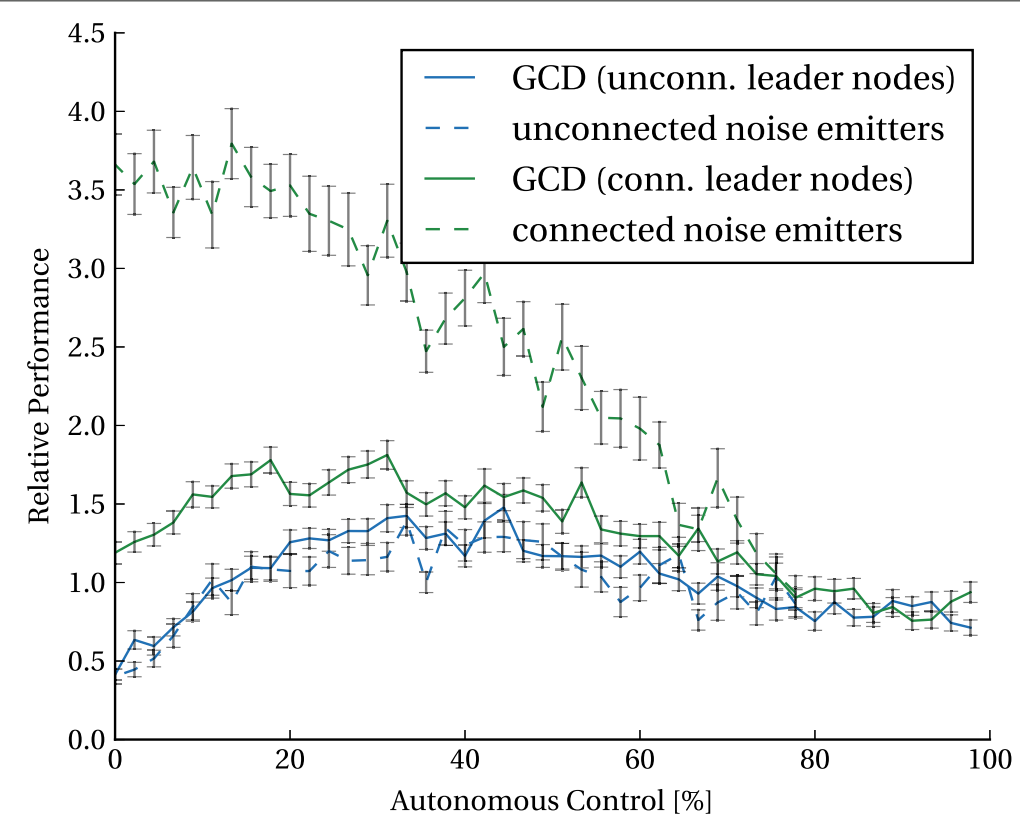

Fig. 5 Relative performance for the GCD model (solid lines) and the derived model of noise emitting leader nodes (dashed lines) shown in comparison. $N=60, \chi=4, s=30$ 
The main qualitative difference between connected and unconnected leader nodes is the decrease of the relative performance with increasing centralized control for the case of unconnected leader nodes. This decrease sets in at around $50-60 \%$ percent connectivity of leader nodes, i.e. when each ring node on average is linked to 2 or 3 leader nodes.

In this range of centralized control, coordination among leader nodes becomes vital and connections among leader nodes reduce systematically incompatible information passed on to ring nodes. In lieu of such coordination ${ }^{4}$, performance starts decreasing again, resulting in an optimum at an intermediate level of leader node connectivity, i.e. for a balance of autonomous and centralized control.

\section{Extension towards realistic performance indicators}

As actual time does not exist in our minimal model of production, so far we cannot distinguish between performance of the scheduling task and the performance of the actual production. The convergence time of the GCD process discussed so far provides only an indirect estimate of logistic performance for a version of autonomous control given a temporal pattern of jobs (represented as a conflict graph) and a set of machines.

In order to bring our model closer to reality, we will employ a 'forward model' strategy ${ }^{5}$. The forward model applied here is described in "Methods" section. Note that the forward model investigation also does no longer depend on performance normalization through reference networks.

If a node did not change its color for $f$ time steps, it is scheduled to be 'produced' on the machine represented by its current color. We call $f$ the 'freezing parameter'. Looking at the performance as a function of $f$ provides qualitative insight into properties of generic scheduling paradigms: At low $f$ the scheduling becomes practically random. At very high $f$, the scheduling requires essentially a fully solved graph with all nodes of the same color being transferred to the corresponding machine almost simultaneously, which again leads to long queues.

The intermediate regime of $f$ allows us to evaluate how the features of the GCD successfully translate into scheduling and production. An example of such a feature is the competition between locally established solution regimes. Figure 6 shows the throughput time as a function of the leader node degree, both for unconnected leader nodes, successfully reproducing the results from Fig. 2. Varying $f$, reveals an interesting shift in peak performance as a function of $f$ : For low values of $f$, low $k_{L}$ attains maximum performance, whereas higher degrees of hierarchical control seem more desirable for long 'freezing times'. Hence, our model provides quantitative evidence for the previously only hypothesized (Trentesaux 2009) understanding that decentralized production control structures are better in situations where scheduling decisions have to be made quickly, whereas more hierarchical structures perform better, when more time is available for the individual decision process.

\section{Discussion}

Within the debate of autonomous control, very few reliable, quantitative facts have emerged from the diverse research directions in algorithmics (Yokoo and Hirayama 1996), applied mathematics (Naor and Stockmeyer 1995; Fraigniaud et al. 2007), statistical physics (Moussaïd et al. 2011; Helbing et al. 2009) and manufacturing research (Zambrano Rey et al. 2014). It has been acknowledged before that minimal models can 


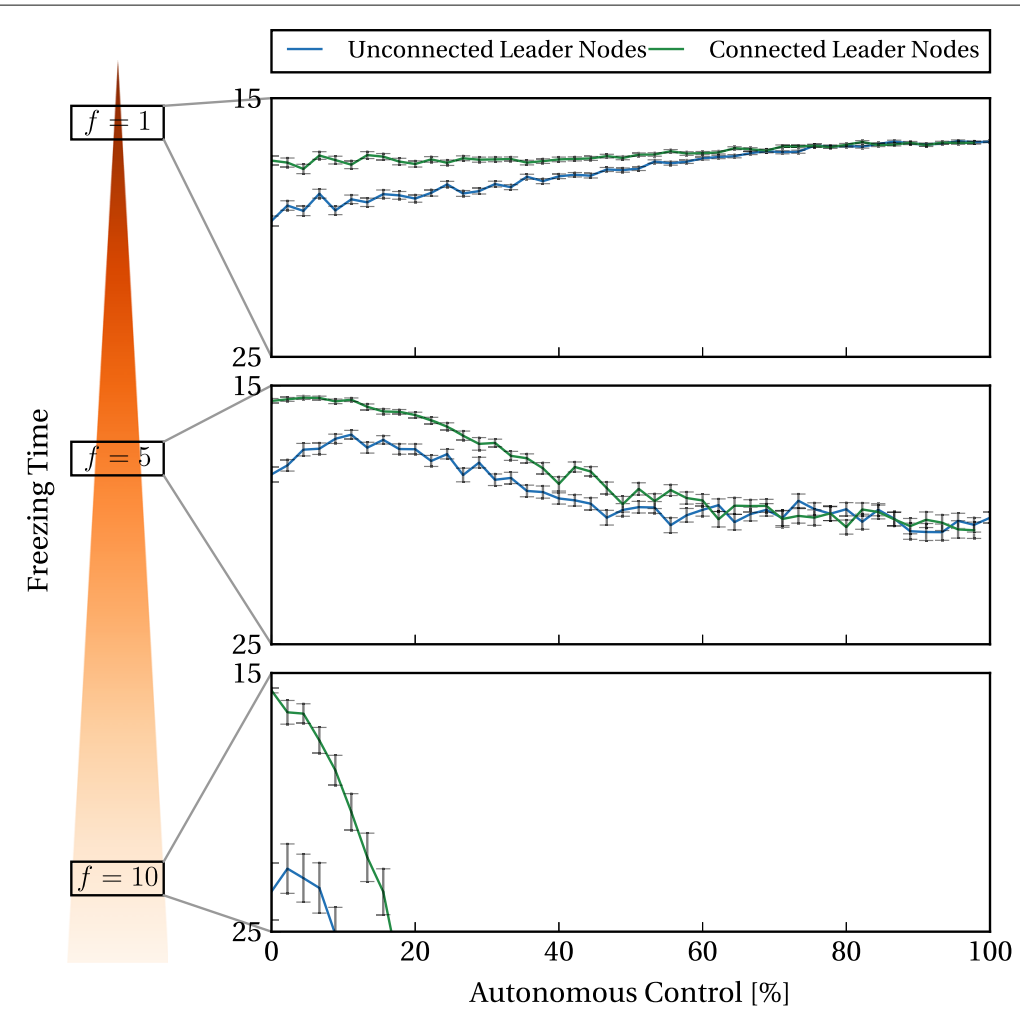

Fig. 6 Makespan of the scheduling problem as a function of the level of autonomous control and freezing time. Makespan is shown on $y$-axis, inverted to reflect notion of performance. $n=60, \chi=4, s=30$

provide some helpful hints towards understanding autonomous control (Windt and Hütt 2010). Here we provide a detailed model study, how the balance of centralized and autonomous control can affect the performance of scheduling and production processes. In our abstract representations of production systems, we find the highest performance at intermediate levels of autonomous control. However, the details matter: (1) The graph's chromatic number regulates the size and shape of this performance peak, (2) connections among leader nodes (i.e. a high level of communication among the agents of centralized control) lead to a strong increase in performance and a shift of maximal performance towards high centralized control. In order to evaluate the relevance of these results for real life applications, we extend the generic framework of GCD to realistic logistic performance indicators (via a forward model extending GCD into the production realm).

Some basic rules of organizing a production process can be derived from the generic findings presented here: (1) Identifying the links between leader nodes with communication in upper management, we see that centralized control that includes such communication outperforms autonomous control alternatives. (2) The chromatic number of a production network or a time pattern of orders can be a useful heuristic indicator of the problem complexity and thus indirectly of the expected logistics performance. (3) Often, a mixture of centralized and autonomous control will lead to the highest logistic performance.

A common understanding of leader nodes is that of an outside source or 'central authority' acting upon the system without adhering to the same set of rules as the nodes 
comprising the system. Here, our leader nodes are part of the system and are only structurally prepared to serve as central authorities. We expected the color dynamics to organize into an information flow, where essentially the leader nodes settle into conflictfree states and then impose compatible states on the ring nodes (thus enslaving the ring nodes and turning into central authorities in the common sense). In contrast, we observe that the self-organized color dynamics turn the 'leader nodes' into noise sources and that this functional role facilitates the convergence towards a conflict-free state.

Among the many possible directions into which this analysis can be extended, three seem most promising.

First, the interplay of performance, balance of centralized and autonomous control and network architecture can be explored. Network science has put forward a huge number of topological identifiers influencing how dynamical processes organize on such networks ${ }^{6}$. Such network properties may also affect the performance resulting from a certain balance of centralized and autonomous control. An additional important question is whether more general network architectures can be associated with the control paradigms discussed here. A detailed analysis of GCD on scalefree graphs (or, in general, graphs with a broad degree distribution) could reveal, whether such categories of nodes (i.e. hubs serving as leader nodes controlling a large number of low-degree 'ring' nodes) emerge under these dynamics.

Second, with the forward model as an interface to real life performance indicators, the development of a novel scheduling tool based on GCD could be envisioned. To this end, extending the work from Hadzhiev et al. (2009) the specific algorithm of GCD (the update scheme and the neighborhood evaluation rule) and its impact on the relationship between performance and control need to be further understood.

Third, the role of information quality in GCD deserves a closer look. Qualitatively speaking, with GCD we see a minimal model implementation of the information mismatch problem mentioned in the Introduction: The incongruous information provided to ring nodes by leader nodes leads to a decrease of performance with increasing centralized control. This aspect deserves a much more detailed analysis in future investigations, distinguishing between three types of information errors: (1) erroneous status perception, (2) time delay of status perception, and (3) true color errors (random color changes). With the distinction of leader nodes and ring nodes in mind, one can also distinguish between status information errors (management is not properly informed) and decision information errors (management does not inform properly).

\section{Conclusion}

The empirical investigation of graph coloring dynamics (Kearns et al. 2006) was an early example of a 'virtual lab' experiment to understand collective problem solving. In (Hadzhiev et al. 2009) cellular automata-like update rules have been developed for the purpose of explaining the counterintuitive findings from Kearns et al. (2006).

Here we argue that the process of graph coloring dynamics, together with the formal representation via cellular automata from Hadzhiev et al. (2009), can serve as a test scenario for understanding performance under variation of the balance between centralized and autonomous control.

Since the early work (Kearns et al. 2006), diverse examples of 'virtual lab' experiments for team performance have been published. 
In Shore et al. (2015) the dependence of the problem solving perfomance on network architecture has been studied in more detail. Recently, in Becker et al. (2017) the impact of social networks on the quality of a collective estimation challenge has been analyzed. In contract to the wide range of studies, where comparatively simple tasks are employed, team performance as a function of team size has recently been studied for a complex task ('crisis mapping', which requires to organize incoming information about crisis events in real time), in order to understand the balance of loss by reduced individual efforts and gain by collaboration (Mao et al. 2016).

How models from statistical physics can be employed to understand some 'stylized facts' about human cooperation and the associated distributed decisions is discussed in (Perc et al. 2017).

Recently, the work by Kearns et al. (2006) was extended towards a large-scale 'virtual lab' experiment, where in addition to human subjects noisy bots (i.e., network nodes controlled by error-prone machines) work included (Shirado and Christakis 2017). The main result was that moderately noisy bots placed in central positions in the network help solve locally unresolvable color conflicts and thus improve global coordination (Shirado and Christakis 2017); see also Gächter (2017). Our numerical results are in line with this observation: The leader nodes in our setup introduce noise enabling ring nodes to consolidate globally incompatible regions of local solutions. Our investigation, however, additionally points to the fact that coordination of the 'noise sources' (i.e., the leader nodes) is of relevance for the global performance.

\section{Materials \& methods}

GCD model

We use Graph Coloring Dynamics (GCD), the agent-based version of the well-studied graph coloring problem. In this setting, $N$ agents play on a network, seeking to choose a color (state) $x$ such that no neighboring agent has the same color. The number of available colors (the size of the action space $\Sigma_{C}$ is limited to $\chi$, the graphs chromatic number. While GCD has been applied to investigate problems of packet switching in shared radio channels (Yeo et al. 2002) and register allocation problems (Smith et al. 2004) and we have previously laid out is applicability to study the effect changing environmental conditions on distributed production planning systems (Windt and Hütt 2010).

The time evolution of the color state vector

$$
\mathbf{x}(t)=\left(x_{1}(t), x_{2}(t), \ldots, x_{n}(t), y_{1}(t), y_{2}(t), \ldots, y_{\chi}(t)\right)
$$

(where $x_{i}(t)$ and $y_{i}(t)$ indicate the color of ring-nodes leader nodes respectively at $t$ ) is generated like the update for a stochastic cellular automaton with asynchronous update. The time evolution requires the specification of two elements: the update scheme (indicating the order, in which nodes are scheduled for update) and the neighborhood evaluation rule (indicating how the new color $x_{i}(t+1)$ is computed from $x_{i}(t)$ and the corresponding neighboring nodes.

For the numerical GCD simulations we follow the 'attention waves with strategic waiting' scheme described in Hadzhiev et al. (2009). When the current color of a node minimizes the number of conflicts in the neighborhood (and is the only color from the color set $\Sigma_{C}$ doing so), the present color state is retained with probability $p$. With probability $1-p$, a color is picked at random from $\Sigma_{C}$. The parameter $p$ thus regulates 
the proportion of 'strategic waiting' in this scheme. Regarding attention waves, we run a simple dynamical process of discrete excitable dynamics (operating on a state space $\Sigma_{E}=\{S, E, R\}$, denoting susceptible, excited and refractory nodes, respectively, where nodes go from excited to refractory $(E \rightarrow R)$ at each time step, go from refractory to susceptible $(R \rightarrow S)$ after a fixed refractory period of two time steps and go from susceptible to excited $(S \rightarrow E)$, when there is an excitation in the direct neighborhood. Additionally, there is a small probability $(f=0.05)$ for spontaneous excitations $(S \rightarrow E)$. At each moment in time, color updates are performed on all excited nodes, thus organizing the color updates to follow 'attention waves' running through the graph based on the excitable dynamics. Such discrete excitable dynamics on graphs have been studied for example in Müller-Linow et al. (2008b); Garcia et al. (2012); Messé et al. (2018).

\section{Networks}

We build upon the investigation and rule space characterization in Hadzhiev et al. (2009). In particular, we focus on strategies featuring 'attention waves' (the passing of ability to change color along network edges) and 'strategic waiting' (the tendency to remain with the current color if it is the sole conflict minimizing color), that have shown to cope well with increasing complexity.

Following the setup in Kearns et al. (2006), we define a ring-graph of $N$ nodes to form the underlying network. Every ring node is connected to its $(\chi-1)$ neighbors on either side, enforcing a chromatic number of $\chi$. A total of $s$ shortcuts is added between ring nodes at random, though maintaining $\chi$-colorability (i.e. when node numbers $i$ are mapped to modulo classes with respect to $\chi, a(i)=i \bmod \chi$, then a shortcut between nodes $i$ and $j$ is allowed if $a(i) \neq a(j)$ ). Finally, we add $\chi$ "leader-nodes" to the network, that are connected to the underlying ring graph through $k_{L}$ links each. We will use the parameter $k_{L}$ to adjust the leader-nodes' impact on the underlying graph. The resulting networks are defined by the tuple $G=G\left(N, l, c, \chi, s, k_{L}, h\right)^{7}$. Typical choices for these parameters are $N=60, l=0$ (no leader nodes) or $l=\chi$ (one leader node per color) and $c=\chi-1$.

\section{Assessment of performance}

A key result of our investigation is the change of performance with the number of leader node links. Performance of graphs with leader nodes is defined as the number of color changes required for solving a graph, compared to a graph without leader nodes, which has the same total number of nodes and non-ring links. Let $r_{C}(G)$ denote the average number of color changes required for solving graph $G$ (with averages performed over many initial color configurations). For graphs with unconnected leader nodes, the performance $P$ can be expressed as ${ }^{8}$

$$
P(G)=\left(\frac{r_{C}\left(G\left(N, \chi, \chi-1, \chi, s, k_{L}, 0\right)\right)}{r_{C}\left(G\left(N+\chi, 0, \chi-1, \chi, s+\chi k_{L}, 0,0\right)\right)}\right)^{-1} .
$$

A performance of $P>1$ means that the graph with leader nodes has been solved with fewer color changes than a reference graph with the same number of nodes and links. The arrangement of $\chi k_{L}$ links as leader node links (implementing a form of hierarchical, centralized control) thus increased the performance compared to a random distribution of these $\chi k_{L}$ as shortcuts in the ring. 
We quantify the performance via this comparison to a reference graph, in oder to eliminate the potential dependence of the runtime (or number of comor changes) on the average node degree (which changes with increasing centralized control).

\section{Information flow}

Time-delayed mutual information is an established measure to assess the flow of information between a sender and a receiver (Kirst et al. 2016). Networks are set up such that the coordination number $c$ of the initial ring determines the network's chromatic number, $c=\chi-1$ (i.e. each node is linked to its $\chi-1$ neighbors in both directions).

As a consequence, out of the $\chi^{n+\chi}$ possible graph state vectors, expressing the color of each ring and leader node as a vector component, only those states are feasible solutions, where the sequence of colors of any $\chi$ neighboring ring nodes or the leader nodes is a permutation of $\Sigma_{C}$. From the $\chi$ ! possible permutations, only $(\chi-1)$ ! are distinct in that they are not circular permutations of each other, i.e. do not correspond to different 'reading frames.' We call these $(\chi-1)$ ! permutations "solution regimes" and their set $\Sigma_{S}^{(0)}$.

$$
\Sigma_{S}^{(0)}=\left\{\sigma_{1}, \sigma_{2}, \ldots, \sigma_{(\chi-1) !}\right\}
$$

The graph is solved if and only if all nodes are assigned to the same solution regime.

In this investigation, we are interested in the binary indicator, if a node is part of a solution regime. A node $i$ is considered to be in a solution regime at time $t$ if and only if the sequence of colors

$$
x_{i}(t), x_{i+1}(t), \ldots, x_{i+(\chi-1)}(t)
$$

corresponds to a solution in $\Sigma_{S}^{(0)}$. In this case, we set $s_{i}(t)=1$. If a color conflict appears in this sequence, $s_{i}(t)=0$.

This framework can now be used to compute the information transfer from, e.g., leader nodes to ring nodes. Let $i$ be a leader node and $j$ a ring node connected to $i$. At each time point $t$, the pair $\left\{s_{i}(t), s_{j}(t)\right\}$ can be computed. On a time window $[t, t+T]$ pair probabilities $p_{c s}\left(c, s \in \Sigma_{C}\right)$, can be estimated, which in turn allow the computation of a mutual information as a measure for the information transfer from leader node $i$ to ring node $j$ :

$$
m=\sum_{c \in \Sigma_{C}, s \in \Sigma_{S}} p_{c s} \log \left(\frac{p_{c s}}{p_{c} p_{s}}\right) .
$$

where $p_{c}$ and $p_{s}$ denote the probabilities of states $c$ in time series $s_{i}$ and $s$ in time series $s_{j}$, respectively.

\section{Simulated annealing model}

To artificially recreate the noise-emitting behavior of leader nodes in the GCD-model, we measure the leader node excitement probability as a function of the number of conflicts in the graph. We then create another network of the same architecture, where the excitement propagation to leader nodes is prevented and leader nodes are artificially excited to consider color changes. Excited leader nodes apply the same decision making strategy as before, so state changes are performed when better configuration is attainable and become increasingly unlikely as the number of conflicts decreases and the master nodes are in a local optimum. In the case of unconnected leader nodes, each leader node is 
excited and may change its color individually, whereas connected leader nodes in this model consider and decide collectively, acting on a wider decision space of all possible solution regimes $\left(\Sigma_{C}=\Sigma_{S}^{(0)}\right)$.

\section{Forward model}

Up to now, the performance analyzed has been an abstract statistical feature, measuring whether the network can be solved with fewer color changes, if the links are arranged according to a centralized control paradigm, rather than a random arrangement. Now we will augment our graph coloring dynamics scheme by a production module, which works along the following set of rules: To assess solutions in terms of schedule quality (rather than just the speed of the solution process), we add a time component to our model: One simulation time unit equals $N$ rounds of the GCD-algorithm. Scheduling is attained as follows:

1 A ring-node (job) that has not changed its color during the previous $f$ time steps will be passed to the machine corresponding to its current color for processing.

2 A node processed in this way will be removed from the graph, i.e. will not be updated or influence the color changing decisions of its neighbors any more.

3 Machines need 1 time step for the processing/production of a job. Additional jobs being queued at that machine during this production time will be delayed accordingly. Jobs are processed one at a time in the sequence of assignment.

Once all nodes (jobs) are transferred into the production process in this way, the total throughput time (i.e. the time until all jobs have been successfully processed) is computed and used as a performance indicator.

The time span between the start time of the first and end time of the last operation (in simulation time units) is considered the makespan of the schedule.

\section{Endnotes}

${ }^{1}$ For ER graphs the diameter scales with $\log N$ (Albert and Barabási 2002), for BA scalefree graphs the diameter scales with $\log \log N$ (Cohen and Havlin 2003).

${ }^{2}$ We claim that the chromatic number is an apt handle to change the problem's complexity.

${ }^{3}$ A naive interpretation of Fig. 2, where connected leader nodes settle first into a conflict-free phase and then force this solution regime upon the rest of the graph, would have led to a much lower frequency of color changes for connected leader nodes, particularly in the later phases.

${ }^{4}$ i.e., for unconnected leader nodes.

${ }^{5}$ See Stephan et al. (2008) for an example from computational neuroscience, where a comparison of experimental data and a stylized model of neuronal excitations is achieved by formulating a model acting upon the simulation results and translating them into a data set with similar statistical features as empirical observations.

${ }^{6}$ Examples include modularity, degree correlations, hierarchy and non-random subgraph compositions.

${ }^{7}$ with $N$, the number of ring nodes; $l$, the number of leader nodes; $c$, the coordination number on the ring (i.e. the number of neighbors to the left and to the right each ring node is connected to); $\chi$, the graphs chromatic number; $s$, the number of shortcuts in the 
ring; $k_{L}$ the degree of the leader nodes and the binary variable $h$ indicating, if the leader nodes form a complete network amongst each other

${ }^{8}$ In the case of interconnected leader nodes, the reference graph would be $G(N+$ $\left.\chi, 0, \chi, \chi, s+\chi k_{L}+\chi(\chi-1) / 2,0,0\right)$.

\section{Additional file}

Additional file 1: As supporting information this file contains a more detailed description of the methods and additional numerical investigations. (PDF $894 \mathrm{~kb}$ )

Acknowledgements

We thank Katja Windt for her support of the development of the idea to use GCD to model scheduling processes.

\section{Authors' contributions}

HB implemented the model and all analyses. DA, JB, and MH developed the forward model and simulated annealing idea. JB and $\mathrm{MH}$ wrote the main manuscript. All authors reviewed the manuscript. HB prepared the manuscript for submission. All authors read and approved the final manuscript.

\section{Competing interests}

The authors declare that they have no competing interests.

\section{Publisher's Note}

Springer Nature remains neutral with regard to jurisdictional claims in published maps and institutional affiliations.

\section{Author details}

${ }^{1}$ RWTH Aachen University, School of Management \& Economics, Kackertstr. 8, 52062 Aachen, Germany. ${ }^{2}$ School of Mathematical and Statistical Sciences, Arizona State University, 85287-1804 Tempe, AZ, USA. ${ }^{3}$ Jacobs University Bremen, Campus Ring 1, 28759 Bremen, Germany.

Received: 4 March 2018 Accepted: 13 June 2018

Published online: 09 July 2018

\section{References}

Albert R, Barabási A-L (2002) Statistical mechanics of complex networks. Rev Mod Phys 74:47-97. https://doi.org/10.1103/ RevModPhys.74.47

Arshad M, Silaghi MC (2004) Distributed simulated annealing. In: Zhang W, Sorge V (eds). Distributed Constraint Problem Solving and Reasoning in Multi-Agent Systems. 112. STM Publishing House, Amsterdam

Becker T, Beber ME, Windt K, Hütt M-T, Helbing D (2011) Flow control by periodic devices: a unifying language for the description of traffic, production, and metabolic systems. J Stat Mech Theory Exp 2011 (05):05004. https://doi.org/10. 1088/1742-5468/2011/05/P05004

Becker J, Brackbill D, Centola D (2017) Network dynamics of social influence in the wisdom of crowds. Proc Natl Acad Sci 201615978

Bikhchandani S, Hirshleifer D, Welch I (1992) A theory of fads, fashion, custom, and cultural change as informational cascades. J Polit Econ 100(5):992-1026

Bornholdt S (2005) Less is more in modeling large genetic networks. Science 310(5747):449-451. https://doi.org/10.1126/ science.1119959. http://www.sciencemag.org/content/310/5747/449.full.pdf

Cohen R, Havlin S (2003) Scale-free networks are ultrasmall. Phys Rev Lett 90:058701. https://doi.org/10.1103/ PhysRevLett.90.058701

de Menezes MA, Barabási A-L (2004) Fluctuations in network dynamics. Phys Rev Lett 92:028701. https://doi.org/10.1103/ PhysRevLett.92.028701

De Werra D, Hertz A (2015) Chromatic scheduling. In: Beineke LW, Wilson RJ (eds). Topics in Chromatic Graph Theory. Encyclopedia of Mathematics and Its Applications, vol. 156, eds. Cambridge University Press. pp 255-276. Chap. 12. https://doi.org/10.1017/CBO9781139519793.015

Drossel B, Schwabl F (1992) Self-organized critical forest-fire model. Phys Rev Lett 69:1629-1632. https://doi.org/10.1103/ PhysRevLett.69.1629

Duffie NA (2008) Challenges in design of heterarchical controls for dynamic logistic systems. In: Dynamics in Logistics. Springer, Berlin. pp 3-24

Duffie N, Fenske J, Vadali M (2013) Coordination of capacity adjustment modes in work systems with autonomous wip regulation. In: Robust Manufacturing Control. Springer, Berlin. pp 135-145

Fabiunke M (1999) Parallel distributed constraint satisfaction. In: Proceedings of the International Conference on Paralle and Distributed Processing Techniques and Applications, PDPTA 1999. CSREA Press. pp 1585-1591. http://citeseerx. ist.psu.edu/viewdoc/download?doi=10.1.1.40.4233\&rep=rep1\&type=pdf

Fraigniaud P, Gavoille C, Ilcinkas D, Pelc A (2007) Distributed computing with advice: Information sensitivity of graph coloring. In: Arge L, Cachin C, Jurdziński T, Tarlecki A (eds). Automata, Languages and Programming. Lecture Notes in Computer Science, vol. 4596. Springer. pp 231-242. https://doi.org/10.1007/978-3-540-73420-8_22

Garcia GC, Lesne A, Hütt M-T, Hilgetag CC (2012) Building blocks of self-sustained activity in a simple deterministic model of excitable neural networks. Front Comput Neurosci 6:50 
Gächter S (2017) Behavioural economics: Occasional errors can benefit coordination. Nature 545(7654):297-298 Graham I, Matthai CC (2003) Investigation of the forest-fire model on a small-world network. Phys Rev E 68:036109. https://doi.org/10.1103/PhysRevE.68.036109

Hadzhiev B, Windt K, Bergholz W, Hütt M-T (2009) A model of graph coloring dynamics with attention waves and strategic waiting. Adv Complex Syst 12(6):549-564. https://doi.org/10.1142/S0219525909002386

Halldórsson MM, Kortsarz G, Shachnai H (2003) Sum coloring interval and k-claw free graphs with application to scheduling dependent jobs. Algorithmica 37:187-209. https://doi.org/10.1007/s00453-003-1031-8

Helbing D, Deutsch A, Diez S, Peters K, Kalaidzidis Y, Padberg-Gehle K, Lämmer S, Johansson A, Breier G, Schulze F, Zerial M (2009) Biologistics and the struggle for efficicency: Concepts and perspectives. Adv Complex Syst 12(6):533-548. https://doi.org/10.1142/S0219525909002374

Hernando L, Mendiburu A, Lozano JA (2016) A tunable generator of instances of permutation-based combinatorial optimization problems. IEEE Trans Evol Comput 20(2):165-179. https://doi.org/10.1109/TEVC.2015.2433680

Hongler M-O, Gallay O, Hülsmann M, Cordes P, Colmorn R (2010) Centralized versus decentralized control—a solvable stylized model in transportation. Physica A: Stat Mech Appl 389(19):4162-4171

Johnson DS, Aragon CR, McGeoch LA, Schevon C (1991) Optimization by simulated annealing: An experimental investigation; part ii, graph coloring and number partitioning. Oper Res 39(3):378-406. https://doi.org/10.1287/opre 39.3.378

Karp RM (1972) Reducibility among combinatorial problems. In: Miller RE, Thatcher JW, Bohlinger JD (eds). Complexity of Computer Computations. The IBM Research Symposia Series. Springer. pp 85-103. https://doi.org/10.1007/978-14684-2001-2_9

Kearns M, Suri S, Montfort N (2006) An experimental study of the coloring problem on human subject networks. Science 313(5788):824-827. https://doi.org/10.1126/science.1127207. http://www.sciencemag.org/content/313/5788/824.full.pdf

Kirst C, Timme M, Battaglia D (2016) Dynamic information routing in complex networks. Nat Commun 7:1 1061. https:// doi.org/10.1038/ncomms11061

Lämmer S, Helbing D (2008) Self-control of traffic lights and vehicle flows in urban road networks. J Stat Mech Theory Exp 2008(04):04019. https://doi.org/10.1088/1742-5468/2008/04/P04019

Mao A, Mason W, Suri S, Watts DJ (2016) An experimental study of team size and performance on a complex task. PloS ONE 11(4):0153048

Marr C, Hütt M-T (2006) Similar impact of topological and dynamic noise on complex patterns. Phys Lett A 349(5):302-305. https://doi.org/10.1016/j.physleta.2005.08.096

Marr C, Hütt M-T (2009) Outer-totalistic cellular automata on graphs. Phys Lett A 373(5):546-549. https://doi.org/10.1016/ j.physleta.2008.12.013

Marx D (2004) Graph coloring problems and their applications in scheduling. Period Polytech Electrial Eng 48(1-2):11-16

Matous P, Todo Y (2017) Analyzing the coevolution of interorganizational networks and organizational performance: Automakers' production networks in japan. Appl Netw Sci 2(1):5

Messé A, Hütt M-T, Hilgetag CC (2018) Toward a theory of coactivation patterns in excitable neural networks. PLoS Comput Biol 14(4):1006084

Meyer M, Hütt M-T, Bendul JC (2015) The elementary flux modes of a manufacturing system: a novel approach to explore the relationship of network structure and function. Int J Prod Res 16. https://doi.org/10.1080/00207543.2015.1106612. http://www.tandfonline.com/doi/pdf/10.1080/00207543.2015.1106612

Moreira AA, Mathur A, Diermeier D, Amaral LA (2004a) Efficient system-wide coordination in noisy environments. Proc Natl Acad Sci U S A 101(33):12085-12090

Moreira AA, Mathur A, Diermeier D, Amaral LAN (2004b) Efficient system-wide coordination in noisy environments. Proc Natl Acad Sci U S A 101(33):12085-12090. https://doi.org/10.1073/pnas.0400672101. http://www.pnas.org/content/101/33/12085.full.pdf+html

Mott KA, Peak D (2006) Stomatal patchiness and task-performing networks. Ann Bot 99(2):219-226. https://doi.org/10. 1093/aob/mcl234

Moussaïd M, Helbing D, Theraulaz G (2011) How simple rules determine pedestrian behavior and crowd disasters. Proc Natl Acad Sci 108(17):6884-6888. https://doi.org/10.1073/pnas.1016507108. http://www.pnas.org/content/108/17/6884.full.pdf+html

Mula J, Poler R, García-Sabater JP, Lario FC (2006) Models for production planning under uncertainty: A review. Int J Prod Econ 103(1):271-285. https://doi.org/10.1016/j.ijpe.2005.09.001

Müller-Linow M, Hilgetag CC, Hütt M-T (2008a) Organization of excitable dynamics in hierarchical biological networks. PLoS Comput Biol 4(9):1000190. https://doi.org/10.1371/journal.pcbi.1000190

Müller-Linow M, Hilgetag CC, Hütt M-T (2008b) Organization of excitable dynamics in hierarchical biological networks. PLoS Comput Biol 4(9):1000190

Myszkowski PB (2008) Solving scheduling problems by evolutionary algorithms for graph coloring problem. In: Xhafa F, Abraham A (eds). Metaheuristics for Scheduling in Industrial and Manufacturing Applications. Studies in Computational Intelligence, vol. 128. Springer. pp 145-167. https://doi.org/10.1007/978-3-540-78985-7_7

Naor M, Stockmeyer L (1995) What can be computed locally?. SIAM J Comput 24(6):1259-1277. https://doi.org/10.1137/ S0097539793254571. http://dx.doi.org/10.1137/S0097539793254571

Pardalos PM, Mavridou T, Xue J (1999) The graph coloring problem: A bibliographic survey. In: Du D-Z, Pardalos P (eds). Handbook of Combinatorial Optimization. Springer. pp 1077-1141. https://doi.org/10.1007/978-1-4613-0303-9_16

Peak D, West JD, Messinger SM, Mott KA (2004) Evidence for complex, collective dynamics and emergent, distributed computation in plants. Proc Natl Acad Sci U S A 101(4):918-922. https://doi.org/10.1073/pnas.0307811100. http://www.pnas.org/content/101/4/918.full.pdf

Perc M, Jordan JJ, Rand DG, Wang Z, Boccaletti S, Szolnoki A (2017) Statistical physics of human cooperation. Phys Rep 687(2017):1-51

Perera S, Bell MG, Bliemer MC (2017) Network science approach to modelling the topology and robustness of supply chain networks: a review and perspective. Appl Netw Sci 2(1):33 
Philipp T, Böse F, Windt K (2006) Evaluation of autonomously controlled logistic processes. In: Proceedings of 5th CIRP International Seminar on Intelligent Computation in Manufacturing Engineering. CIRP, The International Academy for Production Engineering, Ischia. pp 347-352. https://www.journals.elsevier.com/procediacirp/

Pinedo ML (2012) Scheduling: Theory, Algorithms, and Systems. Springer, Berlin. https://doi.org/10.1007/978-1-46142361-4

Rosvall M, Trusina A, Minnhagen P, Sneppen K (2005) Networks and cities: An information perspective. Phys Rev Lett 94(2):028701

Sales-Pardo M, Diermeier D, Amaral LAN (2013) The impact of individual biases on consensus formation. PloS ONE 8(5):58989. https://doi.org/10.1371/journal.pone.0058989

Scholz-Reiter B, Frazzon EM, Makuschewitz T (2010) Integrating manufacturing and logistic systems along global supply chains. CIRP J Manuf Sci Technol 2(3):216-223

Scholz-Reiter B, Freitag M (2007) Autonomous processes in assembly systems. CIRP Ann - Manuf Technol 56(2):712-729. https://doi.org/10.1016/j.cirp.2007.10.002

Scholz-Reiter B, Freitag M, de Beer C, Jagalski T (2005) Modelling dynamics of autonomous logistic processes: Discrete-event versus continuous approaches. CIRP Ann - Manuf Technol 54(1):413-416. https://doi.org/10.1016/ S0007-8506(07)60134-6

Scholz-Reiter B, Görges M, Philipp T (2009) Autonomously controlled production systems - influence of autonomous control level on logistic performance. CIRP Ann - Manuf Technol 58(1):395-398. https://doi.org/10.1016/j.cirp.2009.03. 011

Schreyögg G, Sydow J (2010) Crossroads - organizing for fluidity? dilemmas of new organizational forms. Organ Sci 21(6):1251-1262. https://doi.org/10.1287/orsc.1100.0561. http://pubsonline.informs.org/doi/pdf/10.1287/orsc.1100.0561

Schuh G, Potente T, Varandani R, Hausberg C, Fränken B (2014) Collaboration moves productivity to the next level. Procedia CIRP 17:3-8

Shirado H, Christakis NA (2017) Locally noisy autonomous agents improve global human coordination in network experiments. Nature 545(7654):370-374

Shore J, Bernstein E, Lazer D (2015) Facts and figuring: An experimental investigation of network structure and performance in information and solution spaces. Organ Sci 26(5):1432-1446

Smith MD, Ramsey N, Holloway G (2004) A generalized algorithm for graph-coloring register allocation, vol. 39. ACM, New York. pp 277-288. https://doi.org/10.1145/996893.996875. http://doi.acm.org/10.1145/996893.996875

Stephan KE, Kasper L, Harrison LM, Daunizeau J, den Ouden HEM, Breakspear M, Friston KJ (2008) Nonlinear dynamic causal models for fmri. Neurolmage 42(2):649-662. https://doi.org/10.1016/..neuroimage.2008.04.262

Tang Y, Gao H, Kurths J (2014) Distributed robust synchronization of dynamical networks with stochastic coupling. Circ Syst I Regular Papers, IEEE Trans 61(5):1508-1519. https://doi.org/10.1109/TCSI.2013.2285699

Trentesaux D (2009) Distributed control of production systems. Eng Appl Artif Intell 22(7):971-978. https://doi.org/10. 1016/j.engappai.2009.05.001. Distributed Control of Production Systems

Vázquez A, Pastor-Satorras R, Vespignani A (2002) Large-scale topological and dynamical properties of the internet. Physical Review E 65(6):066130

Watts DJ (2002) A simple model of global cascades on random networks. Proc Natl Acad Sci 99(9):5766-5771. https://doi. org/10.1073/pnas.082090499. http://www.pnas.org/content/99/9/5766.full.pdf+html

Watts DJ, Strogatz SH (1998) Collective dynamics of 'small-world' networks. Nature 393(6684):440-442. https://doi.org/10. 1038/30918

Windt K, Böse F, Philipp T (2008) Autonomy in production logistics: Identification, characterisation and application. Robot Comput Integr Manuf 24(4):572-578. https://doi.org/10.1016/j.rcim.2007.07.008. ICMR2005: Third International Conference on Manufacturing Research

Windt K, Hütt M-T (2010) Graph coloring dynamics: A simple model scenario for distributed decisions in production logistics. CIRP Ann - Manuf Technol 59(1):461-464. https://doi.org/10.1016/j.cirp.2010.03.082

Wolfram S (1984) Cellular automata as models of complexity. Nature 311(5985):419-424. https://doi.org/10.1038/ $311419 \mathrm{a} 0$

Yang S, Ong Y-S, Jin Y (eds) (2007) Evolutionary Computation in Dynamic and Uncertain Environments. Springer. https:// doi.org/10.1007/978-3-540-49774-5

Yeo J, Lee H, Kim S (2002) An efficient broadcast scheduling algorithm for tdma ad-hoc networks. Comput Oper Res 29(13):1793-1806. https://doi.org/10.1016/S0305-0548(01)00057-0

Yokoo M, Hirayama K (1996) Distributed breakout algorithm for solving distributed constraint satisfaction problems. In: Proceedings of the Second International Conference on Multi-Agent Systems. pp 401-408. http://citeseerx.ist.psu. edu/viewdoc/summary?doi=10.1.1.21.65

Zambrano Rey G, Bonte T, Prabhu V, Trentesaux D (2014) Reducing myopic behavior in fms control: A semi-heterarchical simulation-optimization approach. Simul Model Pract Theory 46(0):53-75. https://doi.org/10.1016/j.simpat.2014.01. 005. Simulation-Optimization of Complex Systems: Methods and Applications 